\title{
Finding Sami Cognates with a Character-Based NMT Approach
}

\author{
Mika Hämäläinen \\ Department of Digital Humanities \\ University of Helsinki \\ mika.hamalainen@helsinki.fi
}

\author{
Jack Rueter \\ Department of Digital Humanities \\ University of Helsinki \\ jack.rueter@helsinki.fi
}

\begin{abstract}
We approach the problem of expanding the set of cognate relations with a sequence-tosequence NMT model. The language pair of interest, Skolt Sami and North Sami, has too limited a set of parallel data for an NMT model as such. We solve this problem on the one hand, by training the model with North Sami cognates with other Uralic languages and, on the other, by generating more synthetic training data with an SMT model. The cognates found using our method are made publicly available in the Online Dictionary of Uralic Languages.
\end{abstract}

\section{Introduction}

Sami languages have received a fair share of interest in purely linguistic study of cognate relations. Although various schools of Finno-Ugric studies have postulated contrastive interpretations of where the Sami languages should be located within the language family, there is strong evidence demonstrating regular sound correspondence between Samic and Balto-Finnic, on the one hand, and Samic and Mordvin, on the other. The importance of this correspondence is accentuated by the fact that the Samic might provide insight for second syllable vowel quality, as not all SamicMordvin vocabulary is attested in Balto-Finnic (cf. Korhonen, 1981). The Sami languages themselves (there are seven written languages) also exhibit regular sound correspondence, even though cognates, at times, may be opaque to the layman. One token of cognate relation studies is the Álgu database (Kotus, 2006), which contains a set of inter-Sami cognates. Cognates have applicability in NLP research for low-resource languages as they can, for instance, be used to induce the predicate-argument structures from bilingual vector spaces (Peirsman and Padó, 2010).
The main motivation for this work is to extend the known cognate information available in the Online Dictionary of Uralic Languages (Hämäläinen and Rueter, 2018). This dictionary, at its current stage, only has cognate relations recorded in the Álgu database.

Dealing with true cognates in a non-attested hypothetical proto-language presupposes adherence to a set of sound correlations posited by a given school of thought. Since Proto-Samic is one such language, we have taken liberties to interpret the term cognate in the context of this paper more broadly, i.e. not only words that share the same hypothesized origin in Proto-Samic are considered cognates (hence forth: true cognates), but also items that might be deemed loan words acquired from another language at separate points in the temporal-spatial dimensions. This more permissive definition makes it possible to tackle the problem computationally easier given the limitation imposed by the scarcity of linguistic resources.

Our approach does not presuppose a semantic similarity of the meaning of the cognate candidates, but rather explores cognate possibilities based on grapheme changes. The key idea is that the system can learn what kinds of changes are possible and typical for North Sami cognates with other Uralic languages in general. Taking leverage from this more general level knowledge, the model can learn the cognate features between North Sami and Skolt Sami more specifically.

We assimilate this problem with that of normalization of historical spelling variants. On a higher level, historical variation within one language can be seen as discovering cognates of different temporal forms of the language. Therefore, we want to take the work done in that vein for the first time in the context of cognate detection. Using NMT (neural machine translation) on a character level has been shown to be the single most accurate 
method in normalization by a recent study with historical English (Hämäläinen et al., 2018).

In this paper, we use NMT in a similar character level fashion for finding cognates. Furthermore, due to the limited availability of training data, we present an SMT (statistical machine translation) method for generating more data to boost the performance of the NMT model.

\section{Related Work}

Automatic identification of cognates has received a fair share of interest in the past from different methodological stand points. In this section, we will go through some of these approaches.

Ciobanu and Dinu (2014) propose a method based on orthographic alignment. This means a character level alignment of cognate pairs. After the alignment, the mismatches around the aligned pairs are used as features for the machine learning algorithm.

Another take on cognate detection is that of Rama (2016). This approach employs Siamese convolutional networks to learn phoneme level representation and language relatedness of words. They based the study on Swadesh lists and used hand-written phonetic features and 1-hot encoding for the phonetic representation.

Cognate detection has also been done by looking at features such as semantics, phonetics and regular sound correspondences (St. Arnaud et al., 2017). Their approach implements a general model and language specific models using support vector machine (SVM).

Rama et al. (2017) present an unsupervised method for cognate identification. The method consists of extracting suitable cognate pairs with normalized Levenshtein distance, aligning the pairs and counting a point-wise mutual information score for the aligned segments. New sets of alignments are generated and the process of aligning and scoring is repeated until there are no changes in the average similarity score.

\section{Finding Cognates}

In this section, we describe our proposed approach in finding cognates between North Sami and Skolt Sami. We present the dataset used for the training and an SMT approach in generating more training data.

\subsection{The Data}

Our training data consists of Álgu (Kotus, 2006), which is an etymological database of the Sami languages. From this database, we use all the cognate relations recorded for North Sami to all the other Finno-Ugric languages in the database. This produces a parallel dataset of North Sami words and their cognates in other languages.

The North Sami to other languages parallel dataset consists of 32905 parallel words, of which 2633 items represent the correlations between North Sami and Skolt Sami.

We find cognates for nouns, adjectives, verbs and adverbs recorded in the Giellatekno dictionaries (Moshagen et al., 2013) for North Sami and Skolt Sami. These dictionaries serve as an input for the trained NMT model and for filtering the output produced by the model.

\subsection{The NMT Model}

For the purpose of our research we use OpenNMT (Klein et al., 2017) to train a character based NMT model that will take a Skolt Sami word as its input and produce a potential North Sami cognate as its output. We use the default settings for Open$\mathrm{NMT}^{1}$.

We train a sequence to sequence model with the list of known cognates in other languages as the source data and their North Sami counterparts as the target data. In this way, the system learns a good representation of the target language, North Sami, and can learn what kind of changes are possible between cognates in general. Thus, the model can learn additional information about cognates that would not be present in the North SamiSkolt Sami parallel data.

In order to make the model adapt more to the North Sami-Skolt Sami pair in particular, we continue training the model with only the North Sami-Skolt Sami parallel data for an additional 10 epochs. The idea behind this is to bring the model closer to the language pair of interest in this research, while still maintaining the additional knowledge it has learned about cognates in general from the larger dataset.

\subsection{Using SMT to Generate More Data}

Research in machine translation has shown that generating more synthetic parallel data that can be

\footnotetext{
${ }^{1}$ Version from the project's master branch on the 13 April of 2018
} 
noisy in the source language end but is not noisy in the target end, can improve the overall translations of an NMT model (Sennrich et al., 2015). In light of this finding, we will try a similar idea in our cognate detection task as well.

Due to the limited amount of North Sami-Skolt Sami training data available, we use SMT instead of NMT to train a model that will produce plausible but slightly irregular Skolt Sami cognates for the word list of North Sami words obtained from the Giellatekno dictionaries.

We use Moses (Koehn et al., 2007) baseline ${ }^{2}$ to train a translation model to the opposite direction of the NMT model with the same parallel data. This means translating from North Sami to Skolt Sami. We use the same parallel data as for the NMT model, meaning that on the source side, we have North Sami and on the target side we have all the possible cognates in other languages. The parallel data is aligned with GIZA++ (Och and Ney, 2003).

Since we are training an SMT model, there are two ways we can make the noisy target of all the other languages resemble more Skolt Sami. One is by using a language model. For this, we build a 10-gram language model with KenLM (Heafield et al., 2013) from Skolt Sami words recorded in the Giellatekno dictionaries.

The other way of making the model more aware of Skolt Sami in particular is to tune the SMT model after the initial training. For the tuning, we use the Skolt Sami-North Sami parallel data exclusively so that the SMT model will go more towards Skolt Sami when producing cognates.

We use the SMT model to translate all of the words extracted from the North Sami dictionary into Skolt Sami. This results in a parallel dataset of real, existing North Sami words and words that resemble Skolt Sami. We then use this data to continue the training of the previously explained NMT model for 10 additional epochs.

\subsection{Using the NMT Models}

We use both of the NMT models, i.e. the one without SMT generated additional data and the one with the data separately to assess the difference in their performance. We feed in the extracted Skolt Sami words from the dictionary and translate each word to a North Sami word as it would look like if

\footnotetext{
${ }^{2}$ As described in

http://www.statmt.org/moses/?n=moses.baseline
}

there were a cognate for that word in North Sami.

The approach produces many non-words which we filter out with the North Sami dictionary. The resulting list of translated words that are actually found in the North Sami dictionary are considered to be potential cognates found by the method.

\section{Results and Evaluation}

In this section, we present the results of both of the NMT models, the one without SMT generated data and the one with generated data. The results shown in Table 1 indicate that the model with the additional SMT generated data outperformed the other model. The evaluation is based on a 200 randomly selected cognate pairs output by the models. These pairs have then been checked by an expert linguist according to principles outlined in (4.1)

\begin{tabular}{|l|c|c|}
\hline & NMT & NMT + SMT \\
\hline accuracy & $67.5 \%$ & $83 \%$ \\
\hline
\end{tabular}

Table 1: Percentage of correctly found cognates

Table 2 gives more insight on the number of cognates found and how they are represented in the original Álgu database. The results show that while the models have poor performance in finding the cognates in the training data, they work well in extending the cognates outside of the known cognate list.

\begin{tabular}{|l|c|c|}
\hline & NMT & NMT + SMT \\
\hline Same as in Álgu & 75 & 61 \\
\hline $\begin{array}{l}\text { North Sami word in } \\
\text { Álgu but no cognates } \\
\text { with Skolt Sami }\end{array}$ & 211 & 226 \\
\hline $\begin{array}{l}\text { North Sami word in } \\
\text { Álgu with other } \\
\text { Skolt Sami cognates }\end{array}$ & 646 & 577 \\
\hline $\begin{array}{l}\text { North Sami word not } \\
\text { in Álgu }\end{array}$ & 848 & 936 \\
\hline Cognates found in total & $\mathbf{1 7 8 0}$ & $\mathbf{1 8 0 0}$ \\
\hline
\end{tabular}

Table 2: Distribution of cognates in relation to Álgu

As one of the purposes of our work is to help evaluate and develop etymological research, we will conduct a more qualitative analysis of the correctly and incorrectly identified cognates for the better working model, i.e. the one with SMT generated data. This means that the developers should 
be aware not only of the comparative-linguistic rules etymologists use when assessing the regularity of cognate candidates but semantics as well.

In an introduction to Samic language history (Korhonen, 1981: 110-114), the proto-language is divided into 4 separate phases. The first phase involves vowel changes in the first and second syllables $(\ddot{a}-e \gg e-e, u-e \gg o-e, e-\ddot{a} \gg e-\dot{a}, i-e \gg e-e$, etc.) followed by vowel rotation in the first syllable dependent on the quality of the second-syllable vowel $(e-e » e-e$ but $e-\ddot{a} \gg \varepsilon-\dot{a})$. The second phase entails the loss of quantitative distinction for firstsyllable vowels such that high vowels are normalized as short, and non-high first-syllable vowels are normally long $(e-e \gg \bar{e}-e, \varepsilon-\dot{a} \gg \bar{\varepsilon}-\bar{e}$, etc.). The third phase involves a slight vowel shift in the first syllable and vowel split in the second. And finally, the fourth phase introduces diphthongization of non-high vowels in the first syllable $(\bar{e}-e$ ) $i e-e, \bar{\varepsilon}-\bar{e} \gg e \ddot{a}-\bar{e}$, etc.).

In Table 3, below, we provide an approximation of a few hypothesized sound changes for three words that are attested in Balto-Finnic and Mordvin, alike. *käte 'hand; arm' has true cognates in Finnish käsi, Northern Sami giehta, Skolt Sami kiõtt, Erzya ked' and Moksha käd', *tule 'fire' is represented by Finnish tuli, Northern Sami dolla, Skolt Sami toll, and Mordvin tol, while *pesä 'nest' is attested in Finnish pesä, Northern Sami beassi, Skolt Sami pie'ss, Erzya pize, and Moksha piza. The Roman numerals in the table correspond to four separate phases in Proto-Samic.

\begin{tabular}{|l|c|c|c|c|}
\hline & I & II & III & IV \\
\hline 'hand; arm' & käte & kete & kēte & kiete \\
\hline 'fire' & tule & tole è & tolle & tole \\
\hline 'nest' & pesä & pesà & pēsē & peäsē \\
\hline
\end{tabular}

Table 3: Illustration of some vowel correlations in 4 phases of Proto-Samic

In the evaluation, our attention was drawn to the adherence of (143) items to accepted sound correlations while there were (57) candidates that failed in this respect (cf. Korhonen, 1981; Lehtiranta, 2001; Aikio, 2009). Irregular sound correlation can be exemplified in the North Sami word bierdna 'bear' and its counterpart the Skolt Sami word peä'rnn (the ' prime indicates palatalization in Skolt Sami orthography) 'bear cub'. The former appears to represent the word type found in 'hand' North Sami giehta and Skolt Sami kiõtt, whereas the latter represents the word type found in 'nest' North Sami beassi and Skolt Sami pie'ss and 'swamp' North Sami jeaggi and Skolt Sami je $\ddot{a}^{\prime} \check{g} \breve{g}$. Hence, on the basis of the North Sami word bierdna 'bear', one would posit a Skolt Sami form *piõrnn, whereas the Skolt Sami word peä'rnn 'bear cub' would presuppose a North Sami form *beardni. Both types have firm representation in both languages, so it would seem that these borrowings have entered the languages at separate points in the spatio-temporal dimensions.

\subsection{Analysis of the Correct Cognates}

Correct cognates were selected according to two simples principles of similarity. On the one hand, there was the principle of conceptual similarity in their referential denotations (i.e., this refers to future work in semantic relations). On the other hand, a feasible cognate pair candidate should demonstrate adherence or near adherence to accepted sound law theory. The question of adherence versus near adherence indicated here can be directed to concepts of sound law theory, where conceivable irregularities may further be attributed to points in spatio-temporal dimensions (i.e., when and where a particular word was introduced into the lexica of the two languages involved in the investigation).

In the investigation of 200 random cognate pair candidates, 166 cognate pair candidates exhibited conceptual similarity which in some instances surpassed what might have been discovered using a bilingual dictionary. Of the 166 acceptable cognate pairs 131 candidate pairs demonstrated regular correlation to received sound law theory.

Adherence to concepts of sound law theory can be observed in the alignment of the North Sami words čuoika 'mosquito' and ađa 'marrow' with their Skolt Sami counterparts čuõškk and $\tilde{o} \tilde{o} đ$, respectively. Although these words may appear opaque to the layman, and thus this alignment might be deemed dubious at first, awareness of cognate candidates in the Erzya Mordvin seśke 'mosquito' and $u d^{\prime}$ em 'marrow' helps to alleviate initial misgivings.

As may be observed above, North Sami frequently has two-syllable words where Skolt Sami attests to single-syllable words. This relative length correlation between North Sami and Skolt Sami is described through measurement in 
prosodic feet (cf. Koponen and Rueter, 2016). While North Sami exhibits retention of the theoretical stem-final vowel in two-syllable words, Skolt Sami appears to have lost it. In fact, stemfinal vowels in Skolt Sami are symptomatic of proper nouns and participles (derivations). In contrast, two-syllable words with consonant-final stems appear in both North Sami and Skolt Sami, which means we can expect a number of basic verbs attesting to two-syllable infinitives (North Sami bidjat 'put' and Skolt Sami piijjâd) and contract-stem nouns (North Sami guoppar and Skolt Sami kuõbbâr 'mushroom'). Upon inspection of longer words, it will appear that Skolt Sami words are at least one syllable shorter than their cognates in North Sami, which can be attributed to variation in foot development.

Cognate word correlations between North Sami and Skolt Sami can be approached by counting syllables in the lemmas (dictionary forms). Through this approach, we can attribute some word lengths automatically to parts-of-speech, i.e. there is only one single-syllable verb in Skolt Sami $l e e^{\prime} d$ 'be', and it correlates to a single-syllable verb in North Sami leat. Other verbs are therefore two or more syllables in length in both languages. While two-syllable verbs in North Sami correlate with two-syllable verbs in Skolt Sami, multiplesyllable verbs in North Sami usually correlate to Skolt Sami counterparts in an $\mathrm{X}<=>\mathrm{X}-1$ relationship (number of syllables in the language forms, repectively), where North Sami is one orthographical syllable longer.

Short word pairs demonstrate a clear correlation between two-syllable base words in North Sami and single-syllable base words in Skolt Sami, which with the exception of 4 words were all nouns (54 all told). The high concentration of noun attestation for single-syllable cognate nouns in the two languages of investigation is counterbalanced by the representation of verbs in other word-length correlation groups.

Cognate pairs where both North Sami and Skolt Sami attested to two-syllable lemmas were overrepresented by verbs. There were 47 verbs, 22 nouns, 10 adjectives and 1 adverb. This result is symptomatic of lemma-based research. Surprisingly enough, however, the three-to-two syllable correlation between North Sami and Skolt Sami also showed a similar representation: verbs (13), nouns (2) and adverbs (1).
There was one attested correlation for a 5syllable word engelasgiella 'English language' in North Sami and its 3-syllable counterpart in Skolt Sami engglõskiôll. Since we are looking at a compound word with 3-to-2 and 2-to-1 correlations, we can assume that our model is recognizing individual adjacent segments within a larger unit.

Correct cognates do not necessarily require etymologically identical source forms or structure. The recognized cognate pairs represent both recent loan words or possible irregularities in sound law theory (35) and presumably older mutual lexicon (131) (see 4.2, below). They also attest to differed structure and length (i.e., this may also include derivation and compounding). While a majority of the cognate candidate pairs linked words sharing the same derivational level, 11 represented instances of additional derivation in either the North Sami or Skolt Sami word, and 3 recognized instances where one of the languages was represented by a compound word.

\subsection{Analysis of the Incorrect Cognates}

Incorrect cognates often offer vital input for cognate detection development. There are, of course, words pairs that diverge in regard to both accepted sound law theory and semantic cohesion. These pairs have not yet been applied to development. In contrast, word pairs that appear to adhere to sound law theory yet are not matched semantically might be regarded as false friends. These pairs can be potentially useful in further development.

Of 34 semantically non-feasible candidates, 12 stood out as false friends. One such example pair is observed in the North Sami álgu 'beginning' and the Skolt Sami älgg 'piece of firewood'. These two words, it should be noted, can be associated with the Finnish cognates alku 'beginning' and halko 'piece of split firewood [NB! there is a loss of the word initial $h]$ ', respectively. Since the theoretically expected vowel equivalent of the first syllable $a$ in Finnish is $u o$ and $u e$ in North Sami and Skolt Sami, respectively, we might assume that neither word comes from a mutual Samic-Finnic proto-language.

We do not know to what extent random selection has affected our results. Had the first North Sami noun álgu been replaced with its paradigmatic verb álgit 'begin', the Skolt Sami ällğğed, also translated as 'begin', would have shown direct correlation for $a$ and $\ddot{a}$ in North Sami and 
Skolt Sami, respectively. The second noun, meaning 'piece of split firewood', is actually hálgu in North Sami, which simply demonstrates $h$ retention and the possible recognition problems faced in the absence of semantic knowledge.

\subsection{Summary of the Analyses}

The cognate candidates were evaluated according to two criteria: One query checked for conceptual similarity (correct vs incorrect), and the other checked for regularity according to received sound law theory. While the majority $(65 \%)$ of the word pairs evaluated were both conceptually similar and correlated to received sound law theory, an additional $17 \%$ of the candidates represented irregular sound correlation, as indicated by the figures 131 and 35 below, respectively.

\begin{tabular}{|l|c|c|}
\hline & Similar & Dissimilar \\
\hline Regular & 131 & 12 \\
\hline Irregular & 35 & 22 \\
\hline
\end{tabular}

Table 4: Cognate candidate evaluation

The presence of an $11 \%$ negative score for both sound law regularity and conceptual similarity indicates an improvement requirement of at least $6 \%$ before the machine can be considered relevant $(95 \%)$. The $6 \%$ attestation of false friend discovery, however, displays an already existing accuracy in our algorithm.

\section{Conclusions and Future Work}

In this paper, we have shown that using a character-based NMT is a feasible way of expanding a list of cognates by training the model mostly on the cognate pairs for North Sami words in languages other than Skolt Sami. Furthermore, we have shown that an SMT model can be used to generate synthetic parallel data by pushing the model more towards the direction of Skolt Sami by introducing a Skolt Sami language model and tuning the model with Skolt Sami - North Sami parallel data.

In our evaluation, we have only considered the best cognate produced by the NMT model with the idea of one-to-one mapping. However, it is possible to make the NMT model output more than one possible translation. In the future, we can conduct more evaluation for a list of top candidates to see whether the model is able to find more than one cognate for a given word and whether the overall recall can be improved for the words where the top candidate has been rejected by the dictionary check as a non-word.

We have currently limited our research in cognates between Skolt Sami and North Sami where the translation direction of the NMT model has been towards North Sami. An interesting future direction would be to change the translation direction. In addition to that, we are also interested in trying this method out on other languages recorded in the Álgu database.

We are also interested in conducting research that is more linguistic in its nature based on the cognate list produced in this paper. This will shed more light in the current linguistic knowledge of cognates in the Sami languages. The current results of the better working NMT model are released in the Online Dictionary for Uralic Languages $^{3}$.

\section{References}

Ante Aikio. 2009. The Saami Loan Words in Finnish and Karelian, first edition. Faculty of Humanities of the University of Oulu, Oulu. Dissertation.

Alina Maria Ciobanu and Liviu P Dinu. 2014. Automatic detection of cognates using orthographic alignment. In Proceedings of the 52nd Annual Meeting of the Association for Computational Linguistics (Volume 2: Short Papers), volume 2, pages 99-105.

Mika Hämäläinen and Jack Rueter. 2018. Advances in Synchronized XML-MediaWiki Dictionary Development in the Context of Endangered Uralic Languages. In Proceedings of the Eighteenth EURALEX International Congress, pages 967-978.

Mika Hämäläinen, Tanja Säily, Jack Rueter, Jörg Tiedemann, and Eetu Mäkelä. 2018. Normalizing early english letters to present-day english spelling. In Proceedings of the Second Joint SIGHUM Workshop on Computational Linguistics for Cultural Heritage, Social Sciences, Humanities and Literature, pages 87-96.

Kenneth Heafield, Ivan Pouzyrevsky, Jonathan H Clark, and Philipp Koehn. 2013. Scalable modified Kneser-Ney language model estimation. In Proceedings of the 51st Annual Meeting of the Association for Computational Linguistics (Volume 2: Short Papers), volume 2, pages 690-696.

Guillaume Klein, Yoon Kim, Yuntian Deng, Jean Senellart, and Alexander M. Rush. 2017. OpenNMT: Open-Source Toolkit for Neural Machine Translation. In Proc. ACL.

\footnotetext{
${ }^{3}$ http://akusanat.com/
} 
Philipp Koehn, Hieu Hoang, Alexandra Birch, Chris Callison-Burch, Marcello Federico, Nicola Bertoldi, Brooke Cowan, Wade Shen, Christine Moran, Richard Zens, et al. 2007. Moses: Open source toolkit for statistical machine translation. In Proceedings of the 45th annual meeting of the ACL on interactive poster and demonstration sessions, pages 177-180. Association for Computational Linguistics.

Eino Koponen and Jack Rueter. 2016. The first complete scientific skolt sami grammar in english. FUF 63: 254344, pages 254-266. Pp. 261-265.

Mikko Korhonen. 1981. Johdatus lapin kielen historiaan, volume 370 of Suomalaisen Kirjallisuuden Seuran toimituksia. Suomalaisen Kirjallisuuden Seura, Helsinki.

Kotus. 2006. Álgu-tietokanta. Saamelaiskielten etymologinen tietokanta. http://kaino.kotus.fi/algu/.

Juhani Lehtiranta. 2001. Yhteissaamelainen sanasto, second edition, volume 200 of SuomalaisUgrilaisen Seuran toimituksia. SuomalaisUgrilainen Seura, Helsinki.

Sjur N. Moshagen, Tommi A. Pirinen, and Trond Trosterud. 2013. Building an open-source development infrastructure for language technology projects. In Proceedings of the 19th Nordic Conference of Computational Linguistics (NODALIDA 2013); May 22-24; 2013; Oslo University; Norway. NEALT Proceedings Series 16, 85, pages 343-352. Linköping University Electronic Press.

Franz Josef Och and Hermann Ney. 2003. A systematic comparison of various statistical alignment models. Computational Linguistics, 29(1):19-51.

Yves Peirsman and Sebastian Padó. 2010. Crosslingual induction of selectional preferences with bilingual vector spaces. In Human Language Technologies: The 2010 Annual Conference of the North American Chapter of the Association for Computational Linguistics, pages 921-929. Association for Computational Linguistics.

Taraka Rama. 2016. Siamese convolutional networks for cognate identification. In Proceedings of $\mathrm{COL}$ $I N G$ 2016, the 26th International Conference on Computational Linguistics: Technical Papers, pages 1018-1027.

Taraka Rama, Johannes Wahle, Pavel Sofroniev, and Gerhard Jäger. 2017. Fast and unsupervised methods for multilingual cognate clustering. arXiv preprint arXiv:1702.04938.

Rico Sennrich, Barry Haddow, and Alexandra Birch. 2015. Improving neural machine translation models with monolingual data. arXiv preprint arXiv:1511.06709.
Adam St. Arnaud, David Beck, and Grzegorz Kondrak. 2017. Identifying cognate sets across dictionaries of related languages. In Proceedings of the 2017 Conference on Empirical Methods in Natural Language Processing, pages 2519-2528. 